\title{
The Benefits of SGLT2 Inhibitors in Cardiovascular Prevention, Glycemic Control and Weight Loss, in the Treatment of Diabetes
}

\author{
Bruna de Souza Faustino ${ }^{1}$, Alberto dos Reis Costa Junior ${ }^{1}$, \\ Letícia Nascimento Medeiros Bortolon', Larissa Bianca Paiva Cunha de Sá1, \\ Denise Rosso Tenório Wanderley Rocha ${ }^{1}$, Alberto Krayyem Arbex ${ }^{1,2}$ \\ ${ }^{1}$ Division of Endocrinology, IPEMED Medical School, Rio de Janeiro, Brazil \\ ${ }^{2}$ Visiting Scientist of the Harvard School of Public Health, Harvard University, Boston, USA \\ Email: brunasfaustino@hotmail.com
}

Received 29 November 2015; accepted 24 January 2016; published 28 January 2016

Copyright (C) 2016 by authors and Scientific Research Publishing Inc.

This work is licensed under the Creative Commons Attribution International License (CC BY). http://creativecommons.org/licenses/by/4.0/

(c) (i) Open Access

\begin{abstract}
The sodium and glucose co-transporter inhibitors type 2 (SGLT2) comprises a new class of hypoglycemic drugs to control type 2 diabetes mellitus, in an attempt to add new non-existing benefits to the so far arising classes. Regarding this new class of drugs, represented by dapaglifozin, canaglifozin and empaglifozin, it is important to highlight the benefits brought by these medications to combat hyperglycemia with insulin-independent mechanisms that are beyond glucose reduction, such as cardiovascular events prevention, reduction in HbA1c, weight loss and blood pressure lowering. Recently, a relevant study (Empa-Reg) brought hope and set the spotlight on the prevention of cardiac events among diabetic patients, which is the main cause of mortality within this group. However, despite coming out as a good treatment option, SGLT2 inhibitors are under constant clinical research and, as a new drug, it should be carefully carried out regarding the long-term effects of glycosuria and other possible side effects, such as the observed increase in the incidence of bladder, breast cancer and bone fractures, which require further studies. Therefore, these compounds might represent a landmark approach for the treatment of diabetes.
\end{abstract}

\section{Keywords}

Diabetes, SGLT-2 Inhibitor, Glycemic Control, Cardiovascular Risk, Empa-Reg, Side Effects, Weight Loss

How to cite this paper: Faustino, B. de S., Junior, A. dos R.C., Bortolon, L.N.M., de Sá, L.B.P.C., Rocha, D.R.T.W. and Arbex, A.K. (2016) The Benefits of SGLT2 Inhibitors in Cardiovascular Prevention, Glycemic Control and Weight Loss, in the Treatment of Diabetes. Open Journal of Endocrine and Metabolic Diseases, 6, 87-94. 


\section{Introduction}

Diabetes mellitus (DM) is a heterogeneous group of metabolic disorders that cause hyperglycemia as a common effect, which is a result from defects in insulin action, secretion or both. A DM epidemic is underway in the world. In 1985, it was estimated that 30 million adults had diabetes; this number has grown to 135 million in 1995, reached 173 million in 2002, and it is expected to reach the impressive number of 300 million patients by 2030 [1]-[5]. Several studies look after a better understanding of the medical treatment of type 2 diabetes. Therefore, launching new classes of drugs on this market is essential, to help improve the metabolic profile and the quality of life of diabetic patients.

Among new classes of drugs that have recently emerged worldwide it is worth to focus on a thorough description of the "sodium and glucose co-transporter inhibitors type 2" (or simply "SGLT2”), being present in Brazil the Dapagliflozin [4] [6]. Regarding this new class of drugs is important to highlight the benefits brought by this medication to combat hyperglycemia with insulin-independent mechanisms that are beyond glucose reduction, HbA1c reduction, weight loss and lowering blood pressure. It shows to be beneficial when combined with other oral antidiabetic agents or even insulin when diet and exercise fail in reducing HbA1c [7].

\section{Pharmacological Treatment of Diabetes}

Pharmacological treatment of type 2 diabetes mellitus depends on the stage of the disease, the targets to be reached and comorbidities, such as insulin resistance mechanisms, gradual failure of beta cells, the multiple metabolic disorders and micro and macro vascular repercussions that accompany the natural history of the disease. Metformin, excluding its contraindications and if is well tolerated, it is the pharmacological agent of choice to initiate treatment of type $2 \mathrm{DM}$. Depending on glycemic control one second oral agent becomes necessary. Suggested classes of drugs may be: sulfonylureas, DPP-4 inhibitors, GLP-1 analogues, insulin and glitazones.3 A new class of drugs recently arrival to the market are the sodium and glucose co-transporter inhibitors (SGLT2), in the kidney, which also act in reducing glycated hemoglobin and also contributes to weight loss and low blood pressure [1] [6] [8].

\subsection{SGLT2 Inhibitors (Sodium and Glucose Co-Transporters Inhibitors Type 2)}

The SGLT2 inhibitors is independent of insulin action, are effective in reducing glycated hemoglobin, reducing from $0.5 \%$ to $1.5 \%$, promotes weight loss, a low incidence of hypoglycemia, complements the action of other antidiabetic agents and can be used at any diabetes stage. On the other hand, their side effects, such as recurrent urinary tract infection, genital infection, increased hematocrit, fall in blood pressure requires its use in selected patients [3] [4] [9]-[12]. The kidney plays an important role in glucose homeostasis, not only in gluconeogenesis, but also the glucose reabsorption filtered through all SGLTs called carriers. Approximately 180 grams of glucose per day in a healthy adult is filtered and most of this reabsorbed by SGLT, with 1\% being excreted in the urine. The SGLT are a family of membrane proteins that are responsible for glucose transport through the brush border membranes of the renal proximal tubule and throughout the intestinal epithelium [3]-[5] [13]. While there are several different types of SGLTs, the two most studied are the SGLT1 and SGLT2. Specifically in kidney, SGLT2 is predominantly expressed in the anterior segments of the proximal tubule and is responsible by the reabsorption for over $90 \%$ of the filtered glucose, whereas SGLT1 is located in the distal segments, absorbs the remaining. The SGLT2 has become the focus of a great deal of interest in the field of SGLTs inhibitors regarding type 2 diabetes, by blocking the filtered glucose reabsorption and glycosuria leading to improvements in glycemic control. They are also associated with the loss of calories, providing the potential for weight loss. A rare genetic condition that serves as a model for SGLT2 inhibition is familial renal glycosuria. In this disorder, it is not detrimental to the operation of SGLT2 is SLCA2 secondary mutation of the gene, leading to daily urinary excretion up to 100 grams of glucose [3] [4] [9]-[16]. When the isolated proximal tubular cells from patients with normal glucose tolerance and type 2 diabetes have been exposed to hyperglycemic environment, cells of patients with type 2 diabetes expressed significantly more protein SGLT2 and GLUT2, resulting in glucose uptake which was three times higher than in controls [4] [5] [17].

\section{1) Dapagliflozin}

Dapagliflozin (Forxiga $^{\circledR}$; BristolMyers Squibb, New York, NY, USA; AstraZeneca, London, United Kingdom) was the first SGLT2 inhibitor submitted to FDA (December 2010). However, on January 19th, 2012, the FDA 
refused approval of dapagliflozin and issued a response letter requesting "additional clinical data to allow a better assessment of the benefit-risk profile” for dapagliflozin. This includes ongoing clinical trial data and may require information from new clinical trials. This decrease in approval occurred largely due to concern a sign of cancer, especially breast and bladder. Although animal studies dapagliflozin show no evidence of cancer this does not exclude the possibility that elevated levels of glucose in the bladder accelerated growth rate for pre-existing cancers. In clinical trials, most of the patients who had bladder cancer, hematuria had, indicating that some cancers were probably preexistent [3] [7] [14] [18]-[26]. In November 12th of 2012, the European Commission approved the use of dapagliflozin of $10 \mathrm{mg}$ once a day in type 2 diabetes to improve glycemic control as monotherapy, when diet and exercise alone do not provide adequate glycemic control in patients which the use of metformin is considered inappropriate due to intolerance. Dapagliflozin $10 \mathrm{mg}$ once a day was also approved in Europe and combined with metformin therapy, a sulfonylurea (SU) or with insulin ( \pm oral antidiabetic [OADs]), in conjunction with diet and exercise, when these agents do not provide appropriate glycemic control. Dapagliflozin of 2.5, 5, 10, 20 and 50 mg was investigated in Phase III studies; however, only the results of the doses of $5 \mathrm{mg}$ and $10 \mathrm{mg}$ were presented as being the most relevant doses and more likely to be used clinically [3] [4] [23] [24]. Dapagliflozin has an extensive study program Phase III development, which evaluated both the efficacy and the Agent security from a wide range of type 2 diabetic monotherapy, combining therapy with metformin, SU, pioglitazone, metformin and SU, metformin and sitagliptin and as adjunct therapy to insulin (with or without antidiabetic agents). Phase III studies of dapagliflozin also investigated in special populations such as those with renal impairment (estimated glomerular filtration rate [eGFR] 30 to $59 \mathrm{ml} / \mathrm{min} /$ $1.73 \mathrm{~m}^{2}$ ) and those with documented cardiovascular disease and hypertension [3] [4] [23] [24]. Phase III studies have shown that results dapagliflozin was effective in reducing the glycated hemoglobin (HbA1c) as a monotherapy, double and triple therapy with oral agents, as well as with the combination therapy with insulin, with or without oral hypoglycemic agents. Throughout all Phase III studies, dapagliflozin $5 \mathrm{mg}$ and $10 \mathrm{mg}$ once a day, resulted in significant reductions in HbA1c compared to placebo and active comparator. After 12 and 24 weeks of therapy, HbA1c was decreased by $-0.72 \%$ and $-0.77 \%$ with $5 \mathrm{mg}$ dapagliflozin and $-0.85 \%$ to $-0.89 \%$ of 10 mg dapagliflozin, in type 2 diabetic patients inadequately controlled with diet and physical exercise alone, compared to placebo $(-0.18 \%$ to $-0.23 \%$; $=0.001)$. Fasting glucose reductions were apparent in the first week and continued to decrease significantly with $5 \mathrm{mg}$ and $10 \mathrm{mg}$ of dapagliflozin in the twelfth week compared to placebo $(-1.05$ and $-1.17 \mathrm{mmol} / \mathrm{L}$ versus $-0.33 \mathrm{mmol} / \mathrm{L} ; \mathrm{p}=0.005$ and 0.002 , respectively), and the twenty-fourth week compared to placebo $(-1.34$ and $-1.60 \mathrm{mmol} / \mathrm{L}$ versus $-0.23 \mathrm{mmol} / \mathrm{L} ; \mathrm{p}=0.0005$ and 0.0001 , respectively). Although not statistically significant after 12 and 24 weeks of treatment, a higher proportion of patients treated with $5 \mathrm{mg}$ and $10 \mathrm{mg}$ of dapagliflozin reached an HbA1c target of 7\%, compared to placebo (40\% - 44\% and 51\% - 52\% versus 32\%; $\mathrm{p}=$ not significant). After 102 weeks of treatment, $5 \mathrm{mg}$ and $10 \mathrm{mg}$ of dapagliflozin demonstrated clinically significant reductions compared to placebo, in both HbA1c $(-0.71 \%$ and $-0.61 \%$ to $-0.17 \%)$ and $\mathrm{GJ}(-1.08 \mathrm{mmol} / \mathrm{L},-1.50 \mathrm{mmol} / \mathrm{L}$, from $-0.38 \mathrm{mmol} / \mathrm{L})$. A greater number of patients achieved a goal of HbA1c of $7 \%$ with $5 \mathrm{mg}$ and $10 \mathrm{mg}$ of dapagliflozin compared to placebo (34.4\% and $26.2 \%$ versus 19.4\%) [3] [12] [14] [16] [23] [24]. In a 24-week trial 597 patients with non controlled type 2 diabetes (HbA1c 7\% - 10\%) in glimepiride monotherapy were randomized to dapagliflozin or placebo. The mean reduction of HbA1c to placebo versus all dapagliflozin groups $2.5,5$, and $10 \mathrm{mg}$ statistically significant $(0.13 \%$ versus $0.58 \%, 0.63 \%$ and $0.82 \%$, respectively). This was associated with significant reductions in fasting plasma glucose, postprandial plasma glucose and body weight with $5 \mathrm{mg}$ and $10 \mathrm{mg}$ of dapagliflozin compared to control groups, or, 1.18 $\mathrm{mmol} / \mathrm{L}$ and $1.58 \mathrm{mmol} / \mathrm{L}$, versus $0.11 \mathrm{mmol} / \mathrm{L}$ (21.2 $\mathrm{mg} / \mathrm{dL}$, and $28.4 \mathrm{mg} / \mathrm{dL}$ versus $1.98 \mathrm{mg} / \mathrm{dL}) ; 1.78 \mathrm{mmol} / \mathrm{L}$ and $1.94 \mathrm{mmol} / \mathrm{L}$ versus $0.33 \mathrm{mmol} / \mathrm{L}$ (32.0 mg/dL, and $34.9 \mathrm{mg} / \mathrm{dL}$ versus $5.9 \mathrm{mg} / \mathrm{dL}$ ); and $1.56 \mathrm{~kg}$ and $2.26 \mathrm{~kg}$ versus $0.72 \mathrm{~kg}$, respectively. At the end of the study, $30.3 \%$ in the $5 \mathrm{mg}$ dapagliflozin group and $31.7 \%$ in the 10 mg dapagliflozin group had achieved its goal of HbA1c of 7\% versus $13 \%$ in the placebo group. 4, 5, 11 Patients with uncontrolled type 2 diabetes or with high doses of insulin (50 UI/day) and in use of oral sensitizers were randomized to $10 \mathrm{mg}$ or $20 \mathrm{mg}$ of dapagliflozin per day or placebo for 12 weeks. A basal insulin dose was reduced by $50 \%$ in all three groups. The groups of $10 \mathrm{mg}$ and $20 \mathrm{mg}$ of dapagliflozin showed a reduction in HbA1c of $0.61 \%$ and $0.69 \%$ in comparison with an increase of $0.09 \%$ in the placebo group. The mean fasting plasma glucose increased by $0.98 \mathrm{mmol} / \mathrm{L}(17.8 \mathrm{mg} / \mathrm{dl})$ and $0.13 \mathrm{mmol} / \mathrm{L}(2.34 \mathrm{mg} / \mathrm{dl})$ in the placebo 3 group and in the $10 \mathrm{mg}$ of dapagliflozin group, respectively, but decreased by $0.53 \mathrm{mmol} / \mathrm{L}$ ( $9.54 \mathrm{mg} / \mathrm{dl})$ in $20 \mathrm{mg}$ dapagliflozin group. Reductions in postprandial blood glucose with dapagliflozin were also dose-dependent, i.e. $1.90 \mathrm{mmol} / \mathrm{L}(34.4 \mathrm{mg} / \mathrm{dL})$ in $10 \mathrm{mg}$ group and $2.32 \mathrm{mmol} / \mathrm{L}(41.9 \mathrm{mg} / \mathrm{dL})$ in $20 \mathrm{mg}$ dapagliflozin group in 
comparison with an increase of $1.03 \mathrm{mmol} / \mathrm{L}(18.7 \mathrm{mg} / \mathrm{dL})$ placebo group. The urinary excretion of glucose was $1.5 \mathrm{~g} /$ day in placebo group, compared with $83.5 \mathrm{~g} / \mathrm{day}$ and $85.2 \mathrm{~g} / \mathrm{day}$ in $10 \mathrm{mg}$ and $20 \mathrm{mg}$ dapagliflozin groups, respectively. There was a greater reduction in total body weight in $10 \mathrm{mg}$ and $20 \mathrm{mg}$ of dapagliflozin groups compared to placebo group, in other words, $4.5 \mathrm{~kg}$ and $4.3 \mathrm{~kg}$ versus $1.9 \mathrm{~kg}$, respectively [3] [13] [14] [17]. In addition to lowering glucose, SGLT2 inhibitors have several effects that could be beneficial for patients with metabolic syndrome, such as reducing body weight, decreasing blood pressure (more systolic compared with diastolic), and the positive effects in lipid profile. Additionally, significant reductions in uric acid have been reported which may possibly indicate a decreased risk of cardiovascular events. Regarding the benefit in blood pressure levels, all studies of SGLT2 inhibitors to date demonstrated significant reductions in blood pressure with greater reductions observed in systolic blood pressure $(-1.66 \mathrm{mmHg}$ to $-6.9 \mathrm{mmHg})$ than diastolic $(-0.88$ $\mathrm{mmHg}$ to $-3.5 \mathrm{mmHg}$ ). The effects on blood pressure were not dose related and were no accompanied by any noticeable changes in heart rate or blood pressure increases and/or syncope. The analysis of the Phase IIb studies with empagliflozin also showed greater decreses in systolic blood pressure of $13.4 \mathrm{mmHg}$ to $17.0 \mathrm{mmHg}$ among a subgroup of patients with baseline systolic blood pressure $>140 \mathrm{mmHg}$ compared to the overall population. This information is supported from the results of a study with dapagliflozin which also demonstrated more pronounced effects on blood pressure in patients with an initial systolic blood pressure $>140 \mathrm{mmHg}$. These reductions in systolic blood pressure did not correlate with the change in body weight or glycemic control, suggesting that the antihypertensive effects of empagliflozin and other SGLT2 inhibitors are independent of HbA1c or the reduction in body weight. However, controversy around the use of SGLT2 inhibitors produces long-term reductions in blood pressure arising on the basis of results of a long-term study (102 weeks) of dapagliflozin. This long-term study showed some initial reduction of blood pressure seen at 24 weeks; however, it does not control the variations of the use of antihypertensive medications, which becomes a major limitation for the findings of the investigation [4] [23].

\section{2) Canagliflozin}

Canagliflozin (Invokana ${ }^{\circledR}$; Janssen Pharmaceuticals, Inc., Titusville, New Jersey) was the first SGLT2 inhibitor submitted to FDA (approved on 29 March 2013).

Therefore, more five postmarketing studies were requested by the FDA: 1) a cardiovascular outcomes trial; 2) an enhanced pharmacovigilance program to monitor for malignancies, cases of pancreatitis, severe hypersensitivity reactions, photosensitivity reactions, liver abnormalities, and adverse pregnancy outcomes; a bone safety study; and two pediatric studies under the Pediatric Research Equity Act.

The most frequent side effects of Canagliflozin are vulvovaginal candidiasis and urinary tract infections. It is associated with a diuretic effect, so it can cause a reduction in intravascular volume leading to orthostatic or postural hypotension. This may result in symptoms such as dizziness or fainting, more frequent in the first three months of therapy [4] [27].

Phase III study results demonstrated that canagliflozin was effective in reducing HbA1c in monotherapy, double therapy and triple therapy with oral agents, as well as in combination therapy with insulin, with or without Oral antidiabetics. Throughout all Phase III studies, canagliflozin $100 \mathrm{mg}$ and $300 \mathrm{mg}$ once daily, both resulted in significant reductions in HbA1c compared to placebo and active comparator with reduced somewhat larger with canagliflozin $300 \mathrm{mg}$ once a day [4] [27].

After 26 weeks of treatment, canagliflozin $100 \mathrm{mg}$ and $300 \mathrm{mg}$ once a day significantly reduced HbA1c $(-0.77 \%$ and $-1.03 \%$, respectively) in type 2 diabetic patients inadequately controlled on diet and exercise as compared to placebo $(0.14 \%$, $\mathrm{p}<0.001)$. Fasting blood glucose was also significantly decreased by $-1.50 \mathrm{mmol} / \mathrm{L}$ to $-1.90 \mathrm{mmol} / \mathrm{L}$ with both doses of canagliflozin (placebo $=0.50 \mathrm{mmol} / \mathrm{L} ; \mathrm{p}<0.001$ ), resulting in a greater proportion of patients achieving the HbA1c target of $7 \%$ compared with placebo (44.5\% to $62.4 \%$ versus $20.6 \%$; $\mathrm{p}<0.001)$. Among those who carried out a post-meal tolerance test, canagliflozin improved beta-cell function indices. Both doses significantly reduced plasma glucose levels during the glucose tolerance test, in contrast to small increases observed with placebo. Treatment with canagliflozin also resulted in substantial increases in insulin secretion rate sensitivity and beta-cell glucose function, in contrast with light decreases with placebo. However, C-peptide concentrations were almost identical before and after treatment in all groups [4] [27].

The effectiveness of canagliflozin was sustained when added to metformin. Canagliflozin $100 \mathrm{mg}$ or $300 \mathrm{mg}$ once daily added to metformin has been found not being less than the glimepiride and the combination of metformin and canagliflozin $300 \mathrm{mg}$ once per day provided greater reductions in HbA1c compared to glimepiride $(-0.93 \%$ versus $-0.81 \%$; $95 \%$ CI: $-0.22,-0.02)$. Numerically greater reductions in fasting glucose levels were 
observed after 52 weeks at both doses of canagliflozin compared with glimepiride $(-1.35 \mathrm{mmol} / \mathrm{L}$ of -1.53 $\mathrm{mmol} / \mathrm{L}$ versus $-1.02 \mathrm{mmol} / \mathrm{L}$ ), and while canagliflozin showed sustained decreases throughout the treatment period, there were increases with glimepiride after 18 weeks. Although achieving higher reductions in HbA1c and fasting glycemia with canagliflozin, a similar proportion of patients achieved a HbA1c less than 7\% with canagliflozin or glimepiride when added to the metformin ( $53.6 \%-60.1 \%$ versus $55.8 \%$, respectively). Canagliflozin $100 \mathrm{mg}$ or $300 \mathrm{mg}$ once daily added to patients not controlled on metformin and a SU showed significant reductions in HbA1c (\% $-0.85 \%$ to -1.06 vs. $-0.13 \%, \mathrm{p}<0.001)$ and fasting glucose $(-1.01 \mathrm{mmol} / \mathrm{L}$ to -1.69 $\mathrm{mmol} / \mathrm{L}$ versus $0.23 \mathrm{mmol} / \mathrm{L} ; \mathrm{p}<0.001$ ) compared to placebo. A higher proportion of patients treated with canaglifozin achieved an HbA1c of 7\% when evaluated against placebo ( $43.2 \%$ to $56.6 \%$ versus $18.0 \%$, p < 0.001 ), as adjunctive therapy with metformin and a $\mathrm{SU}$ and fewer patients required rescue therapy $(1.3 \%$ to $1.9 \%$ vs $12.8 \%, \mathrm{p}<0.001$ ). By comparing canaglifozin $300 \mathrm{mg}$ once daily in combination with metformin and SU to sitagliptin, besides metformin and a SU, canaglifozin $300 \mathrm{mg}$ had a greater reduction in HbA1c compared to sitagliptin $100 \mathrm{mg}$ per day after 52 weeks ( $-1.03 \%$ vs. $0.66 \%$, respectively, $95 \% \mathrm{CI}:-0.50,-0.25)$, and the reductions in fasting glucose $(-1.66 \mathrm{mmol} / \mathrm{L}$ vs. $-0.33 \mathrm{mmol} / \mathrm{L}$ canaglifozin against sitagliptin, respectively; $\mathrm{p}<$ 0.001). In addition, more patients treated with canaglifozin $300 \mathrm{mg}$ once daily has reached an HbA1c of $7 \%$ relative to sitagliptin $100 \mathrm{mg}$ per day (47.6\% versus $35.3 \%$ ), or when added to metformin and a SU. A subset of study patients compared adding canaglifozin, metformin and a SU relative to placebo were included in the analysis, demonstrating that both doses of canaglifozin markedly reduced plasma glucose during the test, while the placebo only resulted in slight decreases. However, as was seen in the subgroup of patients that underwent tolerance test in canaglifozin monotherapy study, the C-peptide levels were almost equal before and after treatment as canaglifozin was added to metformin and SU. In addition, both doses of canaglifozin showed increases beta cell sensitivity to glucose in contrast to a slight decrease in sensitivity observed with placebo [4] [27].

\section{3) Empagliflozin}

Empagliflozin (Jardiance ${ }^{\circledR}$; Boehringer Ingelheim Pharmaceuticals, Inc. (BIPI)) was the third SGLT2 inhibitor submitted to FDA (approved on 04 August 2014) [4].

Empaglifozin is also on current studies regarding associations with metformin, sulfonylureas, pioglitazone, and insulin. For example, empagliflozin and linagliptin (Glyxambi ${ }^{\circledR}$ ) was approved on 02 February 2015 as an adjunct to diet and exercise to improve glycemic control in adults with type 2 diabetes (T2D) when both empagliflozin and linagliptin are appropriate treatments [4] [5].

Empaglifozin is approved for treating type 2 diabetic patients, but not for type 1 patients, those with diabetic ketoacidosis, and individuals with severe renal impairment, including end-stage renal failure [4] [5].

Phase III studies with empaglifozin showed that the drug was effective in reducing HbA1c in monotherapy, double therapy, and in triple therapy with Oral Antidiabetics. The fasting glucose levels also decreased significantly from $-1.69 \mathrm{mmol} / \mathrm{L}$ to $-1.54 \mathrm{mmol} / \mathrm{L}$ with both doses of empaglifozin compared with metformin $(1.44$ $\mathrm{mmol} / \mathrm{L}$ ). The effectiveness of empaglifozin remained when added to metformin. Decreases in HbA1c were similar for both doses of empaglifozin, and better than with sitagliptin (HbA1c with empaglifozin $25 \mathrm{mg}$ per day were significantly greater than $\mathrm{HbA1c}$ with sitagliptin $(\mathrm{FG}=-0.68 \mathrm{mmol} / \mathrm{L})$. The effects of empaglifozin $10 \mathrm{mg}$ or $25 \mathrm{mg}$ per day added to metformin were sustained through 90 weeks and provided greater reductions in HbA1c compared to sitagliptin ( $-0.34 \%$ to $-0.63 \%$ vs. $-0.40 \%)$. Numerically higher decreases in fasting glucose levels were observed after 90 weeks with both doses of empaglifozin when measured against sitagliptin $(-1.18 \mathrm{mmol} / \mathrm{L}$ of $-1.76 \mathrm{mmol} / \mathrm{L}$ versus $0.87 \mathrm{mmol} / \mathrm{L})$ and these effects were maintained throughout the period of treatment [5] [28] [29].

After 12 weeks of the study, during which glucose-lowering therapy was to remain unchanged, the adjusted mean differences in the glycated hemoglobin level between patients receiving empagliflozin and those receiving placebo were -0.54 percentage points $(95 \% \mathrm{CI},-0.58$ to -0.49$)$ in the $10-\mathrm{mg}$ group and $-0.60 \%(95 \% \mathrm{CI},-0.64$ to -0.55$)$ in the 25 -mg group. The adjusted mean differences in the glycated hemoglobin level between patients receiving empagliflozin and those receiving placebo were $-0.42 \%(95 \% \mathrm{CI},-0.48$ to -0.36$)$ and $-0.47 \%(95 \%$ $\mathrm{CI},-0.54$ to -0.41$)$, respectively. Over the course of weeks, the differences were $-0.24 \%$ ( $95 \% \mathrm{CI},-0.40$ to $-0.08)$ and $-0.36 \%(95 \% \mathrm{CI},-0.51$ to -0.20$)$ [29].

\subsection{EMPA-REG: Effective Cardiovascular Prevention on Diabetes}

On September 27, 2015 the New England Journal of Medicine published the results of a randomized, multi- 
centered clinical trial involving 7020 diabetic patients using $10 \mathrm{mg}$ or $25 \mathrm{mg}$ of Empaglifozin, compared to placebo, during 3.1 years. The primary outcome was death due to cardiovascular disease (which is the most relevant concern of a diabetologist, because of its high prevalence among diabetic patients), and other events such as non-fatal MI, non-fatal stroke, and unstable angina [29].

Death due to cardiovascular disease happened in 490 of 4687 patients (10.5\%) using empagliflozin, compared to 282 of 2333 patients (12.1\%) in the placebo group (hazard ratio for empagliflozin was 0.86 ; $95.02 \%$ confidence interval, 0.74 to $0.99 ; \mathrm{P}=0.04$ ). No significant between-group differences in the rates of myocardial infarction or stroke were found, but in the empagliflozin group there were significantly lower rates of death from cardiovascular causes (3.7\%, vs. 5.9\% in placebo group; 38\% relative risk reduction), hospitalization for heart failure (2.7\% and $4.1 \%$, respectively; $35 \%$ relative risk reduction), and death from any cause $(5.7 \%$ and $8.3 \%$, respectively; $32 \%$ relative risk reduction) [29].

Over the course of the study, empagliflozin, as compared with placebo, was associated with small reductions in weight, waist circumference, uric acid level, and systolic and diastolic blood pressure with no increase in heart rate and small increases in both LDL and HDL cholesterol [29].

These convincing results, overcoming all previous expectations, put Empaglifozin in the spotlight of cardiovascular risk prevention, in a parallel impact as Metformin at the UKPDS did before. As a new drug, attention must be given to possible side effects still not known. But the evidence is strong and convincing, and diabetic patients were in need of a medication with such a profile [29].

\section{Important Side Effects}

\section{FDA Warnings}

1) Bone Fractures

As of 09 September 2015 FDA released a drug safety communication regarding the increased risk of bone fractures associated with the use of Canagliflozin and Canagliflozin + Metformin as an association. Fractures may appear as early as 12 weeks after start of the drug. Canagliflozin was also reported to be associated with decreases in bone mineral density at the hip and lower spine. It is important to inform patients about this risk, especially in those at risk of bone disorders. This specific risk has not been reported with Dapagliflozin or Empagliflozin [29].

2) Diabetic Ketoacidosis

On 15 May 2015 FDA issued a warning that SGLT2 Inhibitors were associated with Diabetic Ketoacidosis, a disorder usually seen before only in type 1 diabetic patients. This came as a surprise to clinicians, as Ketoacidosis was never reported before in such an association with type 2 diabetes-cases of hyperosmolar hyperglycemia are the usual clinical presentation [29].

This condition was associated with all 3 available drugs of this class: Dapagliflozin, Canagliflozin and Empagliflozin. The signs and symptoms described were: dyspnea, nausea, vomiting, abdominal pain, confusion, and unusual fatigue or sleepiness. 20 cases were reported between March 2013 to June 6, 2014, meaning that it is not a rare condition [29].

3) Urinary Tract Infections and Genital Infections

SGLT2 Inhibitors are associated with recurrent urinary tract infection and strongly associated with genital infection.

The mechanism of action that leads to an increased amount of glucose in urine leads to higher rates of fungal genital infections of the SGLT2 inhibitors, with a higher proportion in women than men (7.4\% to $25.0 \%$ and 2.5\% to $8.3 \%$, respectively). These genital mycotic infections were mild to moderate in severity, usually treated with antifungal therapies prescribed by health professionals or even own treatment, and $1 \%$ led to discontinuation of therapy. Among the male population under study with canagliflozin, uncircumcised men had a higher risk of developing genital infections (e.g., balanitis/balanoposthitis). In women, genital mycotic infections consisted of vulvitis, vulvovaginal candidiasis, vulvovaginal mycotic infection, vulvovaginitis. There was also a higher prevalence of not dose-related infections of the urinary tract (UTI) (from 2.9\% to 13.3\%) compared to placebo, who responded to standard therapy, and appeared similarly with sitagliptin or pioglitazone, and only $1 \%$ resulted in discontinuation of therapy. Both UTIs and genital infections occurred in the first year of therapy and had a low rate of relapse, 3\%. By further looking for discontinuation of therapy in patients treated with dapagliflozin, all interruptions due to genital infections or UTIs occurred within the first year of therapy without interruption in 
the second year of treatment [3] [4] [10].

Canagliflozin: studies show that after 12 weeks of treatment, no obvious differences in conversion from negative baseline urine bacterial culture to positive culture were observed in canagliflozin group vs. sitagliptin/placebo group ( $4.8 \%$ vs. $3.7 \%$, respectively; $\mathrm{P}=0.76$ ) [27];

Empagliflozin: A 12-week study in 408 treatment-naïve patients with poorly controlled T2DM reported UTIs in $1.2 \%$ of those receiving empagliflozin, and $1.2 \%$ and $1.3 \%$ of those taking placebo and metformin, respectively. Mycotic genital infections were reported in $0.8 \%$ and genital pruritus in $1.2 \%$ of patients receiving empagliflozin, and $0 \%$ for placebo and metformin. The proportions of patients who had adverse events, serious adverse events, and adverse events leading to the discontinuation of a study drug were similar in the empagliflozin group and the placebo group. Genital infection was reported in a higher percentage of patients in the pooled empagliflozin group [29].

\section{Conclusion}

The inhibition of SGLT2 is emerging as a common sense and also as an elegant mechanism for delaying diabetes attack in patients who were treated with conventional medicines. These drugs seem to have similar benefits and risks within the class, including significant reductions in HbA1c and fasting glucose and an increased risk of certain types of infections. The most intriguing, perhaps, is its ability to positively influence other important factors, including body weight, blood pressure, lipids and uric acid. Older agents have typically been filled with unfavorable effects on body weight (SU, thiazolidinediones, insulin), on the cardiovascular system (SU and TZDs) and lipids (TZDs). There are still unanswered questions about the possible risk for cancer, the durability of these agents, and how its favorable metabolic profiles will influence the risk of micro and macro vascular disease. The SGLT2 inhibitors have an independent action of insulin, are effective to promote weight loss, have a low incidence of hypoglycemia, complement the action of other anti-diabetic agents, and can be used regardless of diabetes duration. In this era of obesity, an oral medication that promotes weight loss would be a welcome addition to the arsenal of diabetes. However, due to the side effects of repeated urinary tract infections, genital infections, increased hematocrit, and decreased blood pressure, patient selection appears to be important. Despite being a promising medication, SGLT2 inhibitors have many ongoing clinical trials, and should be observed regarding the long-term effects of glycosuria and other potential effects, especially given the observed increase in the incidence of bladder cancer, breast cancers and bone fractures. This new class represents a promising approach for the treatment of diabetes.

\section{Acknowledgements}

We would like to thank Prof. Dr. Aline Marcadenti for her unique dedication to research.

We thank IPEMED Brazil for supporting continuing medical education.

\section{References}

[1] Vilar, L., et al. (2013) Endocrinologia Clínica. 5th Edition, Guanabara Koogan, Rio de Janeiro, 617-675.

[2] American Diabetes Association (2014) Standards of Medical Care in Diabetes 2014. Diabetes Care, 37. http://dx.doi.org/10.2337/dc14-s014

[3] Kim, Y., et al. (2012) Clinical Potential of Sodium-Glucose Cotransporter 2 Inhibitors in the Management of Type 2 Diabetes. Dovepress, 5, 313-327.

[4] Rosenwasser, R.F., et al. (2013) SGLT2 Inhibitors and Their Potential in the Treatment of Diabetes. Dovepress, 6, 453-467.

[5] Mcgill, J.B. (2014) The SGLT2 Inhibitor Empagliflozin for the Treatment of Type 2 Diabetes Mellitus: A Bench to Beside Review. Diabetes Therapy, 5, 43-63. http://dx.doi.org/10.1007/s13300-014-0063-1

[6] AC Farmacêutica (2014) Diretrizes da Sociedade Brasileira de Diabetes: 2013-2014/Sociedade Brasileira de Diabetes; [Organização José Egídio Paulo de Oliveira, Sérgio Vencio]. AC Farmacêutica, São Paulo.

[7] Orme, M., et al. (2014) A Systematic Review and Mixed-Treatment Comparison of Dapagliflozin with Existing AntiDiabetes Treatments for Those with Type 2 Diabetes Mellitus Inadequately Controlled by Sulfonylurea Monotherapy. Diabetology \& Metabolic Syndrome, 6, 73. http://dx.doi.org/10.1186/1758-5996-6-73

[8] Kronenberg, H.M., et al. (2010) Williams Tratado de Endocrinologia. Elsevier, Rio de Janeiro.

[9] Ferrannini, E., et al. (2013) Metabolic Response to Sodium-Glucose Cotransporter 2 Inhibition in Type 2 Diabetic Pa- 
tients. Journal of Clinical Investigation, 124, 499-508. http://dx.doi.org/10.1172/JCI72227

[10] Geerlings, S., et al. (2014) Genital and Urinary Tract Infections in Diabetes: Impact of Pharmacologically-Induced Glucosuria. Diabetes Research and Clinical Practice, 103, 373-381. http://dx.doi.org/10.1016/j.diabres.2013.12.052

[11] Fujita, Y. and Inagaki, N. (2014) Renal Sodium Glucose Cotransporter 2 Inhibitors as a Novel Therapeutic Approach to Treatment of Type 2 Diabetes: Clinical Data and Mechanism of Action. Journal of Diabetes Investigation, 5, 265-275. http://dx.doi.org/10.1111/jdi.12214

[12] Berhan, A. and Barker, A. (2013) Sodium Glucose Co-Transport 2 Inhibitors in the Treatment of Type 2 Diabetes Mellitus: A Meta-Analysis of Randomized Double-Blind Controlled Trials. BMC Endocrine Disorders, 13, 58. http://dx.doi.org/10.1186/1472-6823-13-58

[13] Poudel, R.R. (2013) Renal Glucose Handling in Diabetes and Sodium Glucose Cotransporter 2 Inhibition. Indian Journal of Endocrinology and Metabolism, 17, 588-593. http://dx.doi.org/10.4103/2230-8210.113725

[14] Pérez, L.G., González Albarrán, O. and Cano Megías, M. (2010) Sodium-Glucose Cotransporter 2 (SGLT2) Inhibitors: From Renal Glycosuria to the Treatment of Type 2 Diabetes Mellitus. Nefrología, 30, 618-625.

[15] Osorio, H., Coronel, I., Arellano, A., Pacheco, U., Bautista, R., Franco, M. and Escalante, B. (2012) Sodium-Glucose Cotransporter Inhibition Prevents Oxidative Stress in the Kidney of Diabetic Rats. Oxidative Medicine and Cellular Longevity, 2012, Article ID: 542042. http://dx.doi.org/10.1155/2012/542042

[16] Bays, H. (2013) Sodium Glucose Co-Transporter Type 2 (SGLT2) Inhibitors: Targeting the Kidney to Improve Glycemic Control in Diabetes Mellitus. Diabetes Therapy, 4, 195-220. http://dx.doi.org/10.1007/s13300-013-0042-y

[17] Tahrani, A.A. and Barnett, A.H. (2010) Dapagliflozin: A Sodium Glucose Cotransporter 2 Inhibitor in Development for Type 2 Diabetes. Diabetes Therapy, 1, 45-56. http://dx.doi.org/10.1007/s13300-010-0007-3

[18] Strojek, K., Yoon, K.-H., Hruba, V., Sugg, J., Langkilde, A.M. and Parikh, S. (2014) Dapagliflozin Added to Glimepiride in Patients with Type 2 Diabetes Mellitus Sustains Glycemic Control and Weight Loss over 48 Weeks: A Randomized, Duble-Blind, Parallel-Group, Placebo-Controlled Trial. Diabetes Therapy, 5, 267-283. http://dx.doi.org/10.1007/s13300-014-0072-0

[19] Salvo, M.C., Brooks, A.D. and Thacker, S.M. (2014) Patient Considerations in the Management of Type 2 DiabetesCritical Appraisal of Dapagliflozin. Patient Preference and Adherence, 8, 493-502.

[20] Sun, Y., Zhou, Y., Chen, X., Che, W.-S. and Leung, S.-W. (2014) The Efficacy of Dapagliflozin Combined with Hypoglycemic Drugs in Treating Type 2 Diabetes Mellitus: Meta-Analysis of Randomized Controlled Trials. BMJ Open, 4, e004619.

[21] Bailey, C.J., Gross, J.L., Hennicken, D., Iqbal, N., Mansfield, T.A. and List, J.F. (2013) Dapagliflozin Add-On to Metformin in Type 2 Diabetes Inadequately Controlled with Metiformin: A Randomized, Double-Blind, PlaceboControlled 102-Week Trial. BMC Medicine, 11, 43.

[22] Heerspink, H.J.L., de Zeeuw, D., Wie, L., Leslie, B. and List, J. (2013) Dapagliflozin a Glucose-Regulation Drug with Diuretic Properties in Subjects with Type 2 Diabetes. Diabetes, Obesity and Metabolism, 15, 853-862. http://dx.doi.org/10.1111/dom.12127

[23] Kohan, D.E., Fioretto, P., Tang, W. and List, J.F. (2014) Long-Term Study of Patients with Type 2 Diabetes and Moderate Renal Impairment Shows That Dapagliflozin Reduces Weight and Blood Pressure but Does Not Improve Glycemic Control. Kidney International, 85, 962-971. http://dx.doi.org/10.1038/ki.2013.356

[24] Kilov, G., Leow, S. and Thomas, M. (2013) SGLT2 Inhibition with Dapagliflozin. A Novel Approach for the Management of Type 2 Diabetes. Australian Family Physician, 42, 706-710.

[25] Sun, Y., Zhou, Y., Chen, X., Che, W.-S. and Leung, S.-W. (2013) The Efficacy of Dapagliflozin Combined with Hypoglycemic Drugs in Treating Type 2 Diabetes: Protocol for Meta-Analysis of Randomized Controlled Trials. Systematic Reviews, 2, 103.

[26] Merovci, A., Solis-Herrera, C., Daniele, G., Eldor, R., Fiorentino, T.V., Tripathy, D., et al. (2014) Dapagliflozin Improves Muscle Insulin Sensitivity but Enhances Endogenous Glucose Production. The Journal of Clinical Investigation, 124, 509-514. http://dx.doi.org/10.1172/JCI70704

[27] Kaushal, S., Singh, H., Thangaraju, P. and Singh, J. (2014) Canagliflozin: A Novel SGLT2 Inhibitor for Type 2 Diabetes Mellitus. North American Journal of Medical Science, 6, 107-113.

[28] Ridderstrale, M., Svaerd, R., Zeller, C., Kim, G., Woerle, H.J., Broedl, U.C. and on Behalf of the EMPA-REG H2H-SUTM Trial Investigators (2013) Rationale, Design and Baseline Characteristics of a 4-Year (208-Week) Phase III Trial of Empagliflozin, an SGLT2 Inhibitor, versus Glimepiride as Add-On to Metformin in Patients with Type 2 Diabetes Mellitus with Insufficient Glycemic Control. Cardiovascular Diabetology, 12, 129. http://dx.doi.org/10.1186/1475-2840-12-129

[29] Zinman, B., Wanner, C., Lachin, J.M., Fitchett, D., Bluhmki, E., Hantel, S., et al. (2015) Empagliflozin, Cardiovascular Outcomes, and Mortality in Type 2 Diabetes. The New England Journal of Medicine, 373, 2117-2128. http://dx.doi.org/10.1056/NEJMoa1504720 